\title{
\#NosRelacionamosEnRed. Proyecto de intervención en situaciones de aislamiento social
}

\author{
M. ${ }^{a}$ Carmen Fernández López y Elisa de las Fuentes Gutiérrez
}

Universidad de Alcalá

\begin{abstract}
RESUMEN: Se presenta en este artículo \#NosRelacionamosEnRed, un proyecto de intervención social creado por los grupos GIELEN y ELENyA de la Universidad de Alcalá para estudiar el impacto del confinamiento en el hogar y aislamiento social de aquellos niños y adolescentes que se encontraban en el año 2020 en un proceso incipiente de integración sociolingüística y cultural, y proponer acciones conjuntas con los profesionales de la educación. Se exponen, además, los resultados de la primera fase del proyecto, fruto del análisis de la información generada a través de las redes sociales, y se advierte la necesidad de continuar investigando para encontrar propuestas educativas que ayuden a paliar la posible brecha en la integración que pudiera haberse producido durante el confinamiento en el hogar.
\end{abstract}

PALABRAS CLAVE: Integración sociolingüística, integración cultural, niños, adolescentes, aislamiento social, comunicación.

\section{\#NosRelacionamosEnRed. Project for intervention in situations of social isolation}

ABSTRACT: This article presents \#NosRelacionamosEnRed, a social intervention project created by the GIELEN and ELENyA groups at the University of Alcalá aimed at studying the impact of home confinement and social isolation of children and adolescents, who were in an early process of sociolinguistic and cultural integration in 2020 . Likewise, the project aims at proposing joint actions with education professionals. On the other hand, this article also presents the results of the first phase of the project, derived from the analysis of the information generated through the use of social networks. Finally, it notes the need for further research that seeks to find educational proposals capable of helping to bridge the potential gap in integration that may have occurred during home confinement.

KEYWORDS: Sociolinguistic integration, cultural integration, children, adolescents, social isolation, communication. 


\section{Introducción}

En las siguientes páginas presentamos un proyecto de intervención social titulado \#NosRelacionamosEnRed ${ }^{47}$ desarrollado con el objetivo de estudiar e intervenir en las consecuencias sufridas por el cierre de las aulas y el confinamiento en el hogar motivados por la pandemia ocasionada por el COVID-19. En marzo de $2020^{48}$ con el fin de evitar la propagación del virus y el contagio se impusieron normas de aislamiento y distanciamiento interpersonal que truncaron el desarrollo de las habilidades comunicativas de niños y adolescentes, en general, y el proceso de integración sociolingüística y cultural de alumnado inmigrante de reciente incorporación al centro escolar, en muchos casos con desconocimiento del idioma. El miedo, la precaución y el cuidado del otro se convirtieron en una prioridad, dejando de lado procesos de integración social, de desarrollo de las competencias comunicativas o del logro del bienestar emocional.

La observación del impacto de esta anómala situación entre la población infantil y adolescente, mediante la recogida de información a través de las redes sociales, y el análisis de las respuestas recibidas, permitirían actuar rápidamente en el ámbito educativo y social para paliar los posibles efectos adversos en el desarrollo del escolar. Por ese motivo, la iniciativa se dirigió a docentes y educadores encargados de la formación de niños y adolescentes de 6 a 17 años, y en especial a los que tenían en sus aulas alumnos inmigrantes de reciente incorporación al contexto escolar, que se encontraban en un proceso incipiente de integración sociolingüística y cultural antes de que se cerraran las aulas a causa del COVID-19.

El proyecto se enmarca en las labores que están realizando los grupos de innovación e investigación de la Universidad de Alcalá GIELEN (Grupo de Innovación en Enseñanza de la Lengua Española a Niños) y ELENyA (Grupo de Investigación en Enseñanza de la Lengua Española a Niños y a Adolescentes) con relación a la enseñanza de la lengua española y al estudio de las habilidades y capacidades comunicativas de los niños y adolescentes, sobre todo en contextos escolares, en el marco de INMIGRA3-CM (ref. H2019/HUM-5772), la red de

\footnotetext{
${ }^{47}$ El proyecto se presentó en el Hackathon Virtual de la Comunidad de Madrid celebrado del 4 al 13 de abril de 2020 con el lema \#VenceAlVirus. Se puede acceder al resumen del mismo en el catálogo de proyectos seleccionados publicado por la Consejería de Ciencia, Universidades e Innovación de la Comunidad de Madrid en el siguiente enlace: https://www.madrimasd.org/uploads/proyectos_mejorconsiderados_vfinal.pdf (p. 114). ${ }^{48}$ Real Decreto 463/2020, de 14 de marzo y Real Decreto-ley 10/2020, de 29 de marzo, en los que el Gobierno de España establece el estado de alarma y unas semanas después endurece las medidas del confinamiento a causa del coronavirus.
} 
Grupos de la Comunidad de Madrid que se encarga del estudio de la integración de la población inmigrante desde diferentes perspectivas.
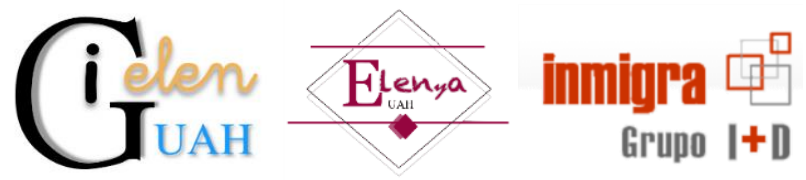

Con motivo del Hackathon Virtual que la Comunidad de Madrid celebró del 4 al 13 de abril de 2020 bajo el lema \#VenceAlVirus, se activó el proyecto \#NosRelacionamosEnRed en uno de los retos planteados por la organización, el reto de Comunidad, para responder a la pregunta lanzada por los creadores del Hackathon: “¿Cómo podemos crear comunidad y cuidar a las personas ante epidemias o situaciones de aislamiento ahora y en los próximos meses?". La investigación que presentamos se planteó con el objetivo de promover acciones educativas conjuntas con los profesionales de los centros docentes y frenar los efectos del COVID-19 en los colectivos más vulnerables, partiendo del núcleo familiar en el que se encontraban confinados los informantes.

Intereses semejantes han movido a otros investigadores, como Rodicio García et al. (2019), que emprendieron una amplia recogida de información entre estudiantes, principalmente universitarios, a través de cuestionarios, preocupados por las consecuencias de la reconocida brecha digital existente en el ámbito educativo. La dificultad con la que cuentan los alumnos al enfrentarse al uso de las nuevas tecnologías puede ser ocasionada por problemas de accesibilidad, sobre todo, en los ámbitos más desfavorecidos, entre los que se encuentra el colectivo de inmigrantes en la mayoría de las ocasiones; o por carecer de competencias digitales para su uso y explotación. Sea el que sea el motivo que ocasiona dicha brecha digital, la situación se ve agravada en situaciones extremas como la que surgió en marzo de 2020 a causa del confinamiento sufrido por la pandemia ocasionada por el COVID19. La mayoría de la población en España cuenta con el equipamiento básico para acceder a internet, y así lo corroboran en su estudio Rodicio García et al.; otra cosa es la capacitación y la competencia para hacer uso de esos recursos con fines educativos. La estrategia nacional de prevención y lucha contra la pobreza y la exclusión social aprobada por el Consejo de Ministros el 22 de marzo de 2019 para su aplicación hasta 2023 (Gobierno de España 2019) se organiza en torno a cuatro metas: combatir la pobreza, inversión social en las personas, protección social ante los riesgos del ciclo vital y eficacia y eficiencia de las políticas. Los flujos migratorios además constituyen un importante factor condicionante en la tasa de riesgo de pobreza infantil (menores de 18 años); así en 2017 el 22 por ciento de los menores residentes en España 
era de origen inmigrante (un 15,3 por ciento contaba con dos progenitores nacidos fuera y un 6,7 descendía de parejas mixtas), y 2 de cada 3 menores con dos progenitores nacidos fuera de la UE (65,4 por ciento) se encontraba en riesgo de pobreza, frente al 22 por ciento de los menores en hogares con dos progenitores nacidos en España (Gobierno de España 2019: 26). En estas condiciones la tasa de abandono educativo temprano y la tasa de repetición en estos grupos resultan ser especialmente elevadas; junto a otras carencias asociadas como el hecho de no participar de las actividades y acontecimientos escolares por los que haya que pagar (viajes, deportes, instrumentos musicales...) o falta de libros adecuados a su edad y otros materiales educativos, entre los que por supuesto se incluyen los que dependen de las nuevas tecnologías (ordenadores, tabletas o móviles). Esta brecha social evidente, agravada con procesos de inmigración, pero no solo afectando a estos grupos poblacionales, no había mejorado sustancialmente cuando surgió en marzo de 2020 la necesidad de reorganizar el sistema de impartición y seguimiento de las clases; el acceso a los materiales docentes a través de las nuevas tecnologías desde los hogares supuso un importante retroceso en la evolución escolar de los grupos más vulnerables por falta de medios y de habilidades para su uso.

Nuestra propuesta de intervención educativa se encuentra en la línea de iniciativas que se van consolidando en los centros escolares basadas en la experimentación con las redes sociales como espacios educativos virtuales en los que se propician interacciones de cooperación e igualdad (Barragán Sánchez y Ruiz Pinto 2013; Muñoz Prieto et al. 2013). La creación de comunidades de aprendizaje basadas en las redes sociales virtuales permite que todos sus miembros accedan fácilmente a la información, compartan recursos y experiencias y, como consecuencia, se creen relaciones interpersonales fluidas cargadas de afectividad.

La implementación de nuestro proyecto se llevó a cabo siguiendo un planteamiento cualitativo de investigación-acción organizado en una primera fase de diagnóstico y análisis de la realidad, seguida del diseño de propuestas de acción educativa, la puesta en práctica y observación de resultados. La recogida de datos e información entre los diferentes grupos de escolares pudo llevarse a cabo con relativa facilidad y rapidez puesto que el confinamiento social y el uso de las redes sociales para la comunicación entre iguales permitió acceder a una muestra representativa en un corto periodo de tiempo. El método etnográfico en el que se fundamentaba el proyecto se evidenció en la recogida de información de carácter situacional, colaborativa y participativa sobre los intercambios comunicativos de los niños y adolescentes durante el confinamiento; se prepararon cuestionarios que fueron difundidos con la mediación del equipo del proyecto a través de 
plataformas gratuitas que provocaban la emisión de mensajes de ánimo, relatos de experiencias y dibujos.

Los cuestionarios que se enviaron a las familias constaban de 14 preguntas organizadas en diferentes bloques: datos personales, relaciones interpersonales y comunicación escrita. En el primer bloque se solicitó el nombre, la edad y lugar donde residían los estudiantes (preguntas 1, 2 y 3); en el segundo bloque, se preguntó por datos más específicos de la investigación, concretamente, por la comunicación entre los estudiantes y las relaciones interpersonales durante la cuarentena a través de las redes sociales (preguntas 4, 5, 7 y 8). Puesto que se pretendía conocer cuáles eran las lenguas de aquellos estudiantes con los que se comunicaban y la suya propia para detectar situaciones de aislamiento, también se solicitaron estos datos personales en el segundo bloque (preguntas 6, 9 y 10). En el tercer bloque, los niños y adolescentes tenían que escribir una carta a uno de sus amigos contándole su experiencia durante el confinamiento y enviándole un mensaje de ánimo. Se facilitaron para ello dos opciones: mensajes escritos en el cuestionario y mensajes escaneados escritos en papel (preguntas 11 y 12).

Las preguntas 13 y 14 se realizaron con el objetivo de poner en funcionamiento el sistema de comunicación entre los participantes. Los destinatarios de las cartas de aquellos estudiantes que habían aceptado su envío recibieron las mismas con un nuevo formato dado por las promotoras del proyecto. A continuación, se muestran las preguntas unificadas de los cuestionarios de los niños y adolescentes y seguidamente, en el apartado 2, algunos ejemplos de los textos que se enviaron a los destinatarios a petición de los participantes. Estos textos han sido anonimizados, por lo que los nombres de los estudiantes y los datos que puedan resultar identificativos son ficticios.

Cuestionario para niños y adolescentes:

1. Escribe tu nombre

2. Escribe tu edad

3. ¿En qué ciudad o pueblo vives?

4. ¿Hablas con tus compañeros del colegio/instituto durante la cuarentena?

5. ¿Qué plataformas o redes sociales utilizas para hablar con ellos?

6. ¿Qué lenguas conocen esos compañeros?

7. ¿Con qué otras personas te relacionas durante la cuarentena?

8. ¿Qué plataformas o redes sociales utilizas para hablar con ellas?

9. ¿Qué lenguas conocen esas personas? 
10. ¿Qué lenguas conoces tú?

11. Opción 1. Piensa en uno de tus amigos y escríbele una carta contándole tu experiencia durante el confinamiento y dándole un mensaje de ánimo. Mira el ejemplo.

12. Opción 2. Si lo prefieres, puedes subir una foto de tu carta escrita a mano y de los dibujos que hagas.

13. ¿Quieres que enviemos tus cartas a otras personas?

14. Si has respondido "sí", escribe aquí el correo electrónico del destinatario (un amigo, un profesor...).

\section{Resultados de la primera fase}

El análisis de la información generada a través de las redes sociales ha permitido corroborar la diversidad lingüística y cultural que caracteriza las aulas y la sociedad en la que se enmarca el estudio ${ }^{49}$. A continuación, se muestran los resultados organizados en los diferentes bloques.

\subsection{Datos personales}

Edad

Se recogieron para su estudio 58 respuestas del cuestionario para niños (6-12 años) y otras 59 del de adolescentes (13-17 años). En la tabla 1 se muestra el número de participantes de cada edad, desde los 6 hasta los 17 años.

\begin{tabular}{|c|c|}
\hline EDAD (años) & PARTICIPANTES \\
\hline 6 & 4 \\
\hline 7 & 8 \\
\hline 8 & 6 \\
\hline 9 & 15 \\
\hline 10 & 15 \\
\hline 11 & 9 \\
\hline 12 & 1 \\
\hline 13 & 10 \\
\hline 14 & 11 \\
\hline 15 & 15 \\
\hline 16 & 14 \\
\hline 17 & 9 \\
\hline
\end{tabular}

Tabla 1. Edad y número de participantes.

\footnotetext{
${ }^{49}$ Algunos resultados del estudio han sido expuestos detenidamente por Jonathan García Navarro en el Trabajo Final de Grado que presentó en la Facultad de Educación de la Universidad de Alcalá en junio de 2020, titulado "Niños, adolescentes y redes sociales: Nueva forma de comunicación".
} 
Sexo

En el estudio participaron 66 mujeres y 51 hombres. A continuación, en el gráfico i se muestra la distribución porcentual de los participantes por sexo.

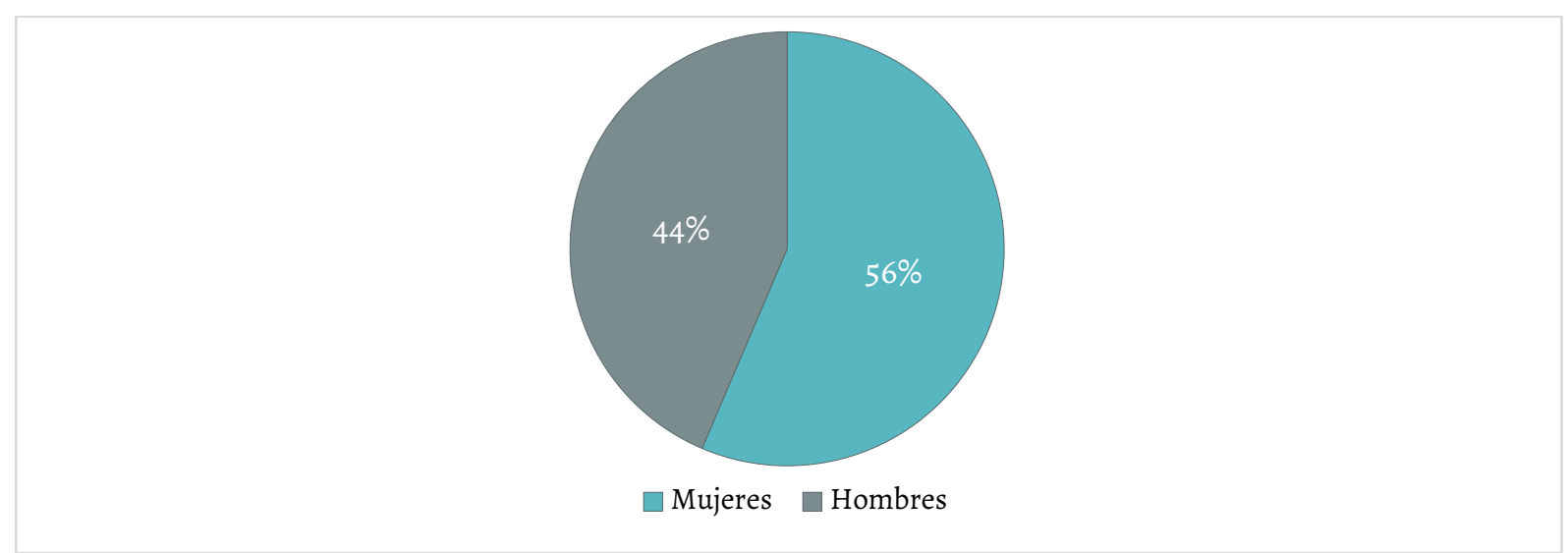

Gráfica 1. Distribución porcentual de los participantes por sexo

Lugar de residencia

La mayoría de los participantes provenía de la zona centro peninsular (95,73\%); concretamente, de la Comunidad de Castilla-La Mancha (provincia de Guadalajara), de la Comunidad de Madrid (Alcalá de Henares, Boadilla del Monte, Madrid, Meco y Torrejón de Ardoz) y de la Comunidad de Castilla y León (Ávila). La difusión a través de las redes sociales permitió recibir cuestionarios cumplimentados de otras zonas de España como Barcelona, Vigo y La Rioja (Logroño) (4,27\%). Cabe destacar que un estudiante informó de que procedía tanto de Barcelona como de un municipio de Madrid, El Escorial, por lo que se ha considerado residente en ambas zonas $(0,85 \%)$.

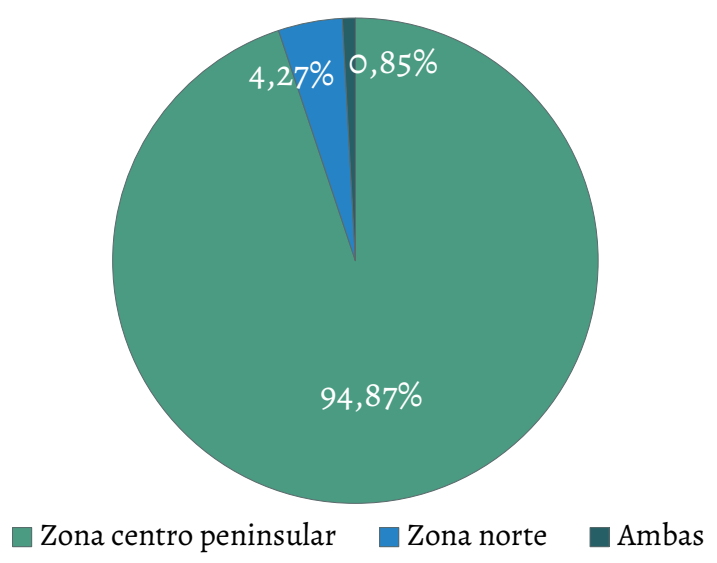

Gráfica 2. Distribución porcentual de los participantes por lugar de residencia 


\section{Dominio de lenguas de los participantes}

En las gráficas 3 y 4 se muestra la gran cantidad de lenguas que nuestros informantes, niños y adolescentes, declaran conocer, además del español.

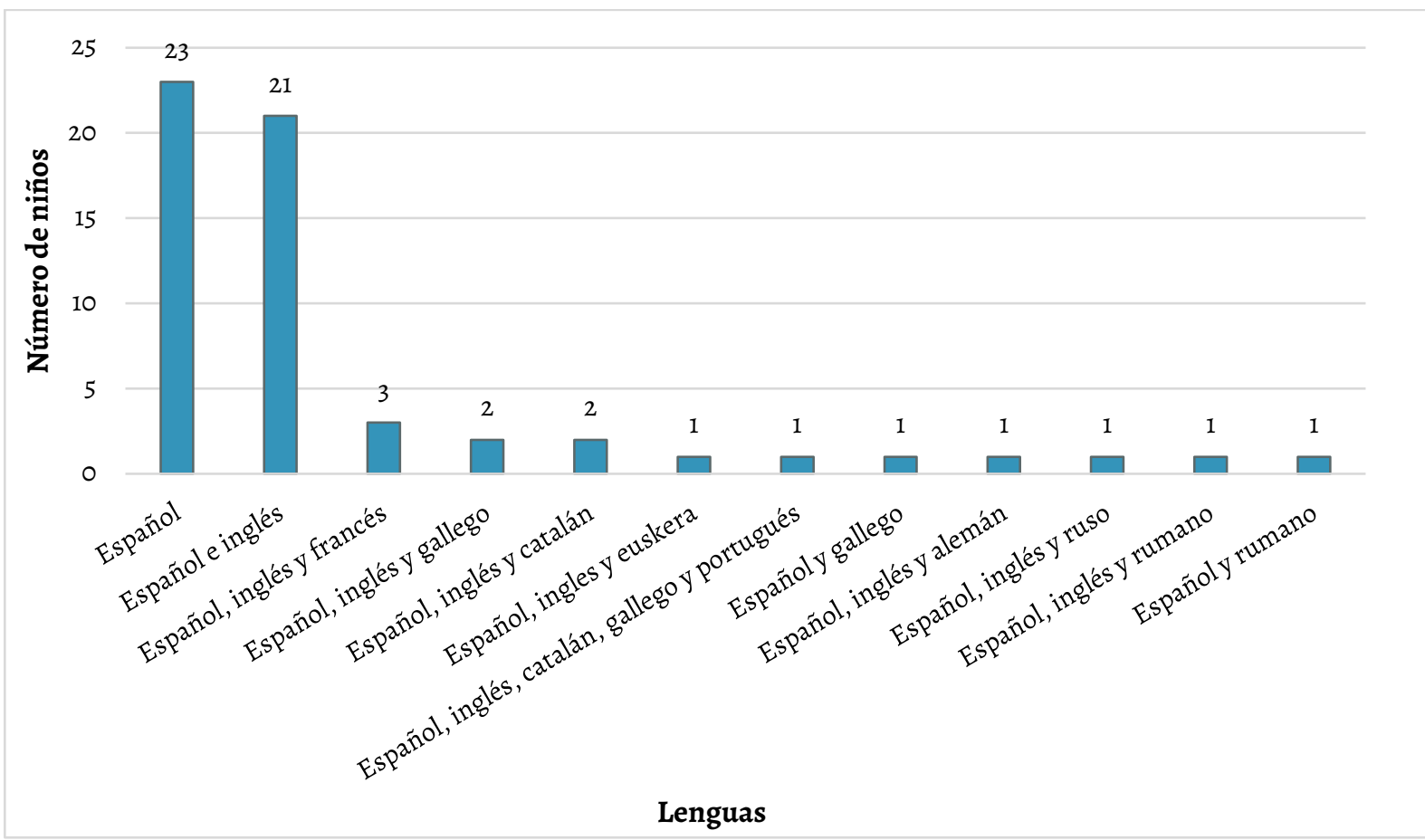

Gráfica 3. Cuestionario para niños. Pregunta: ¿Quélenguas conoces tú?

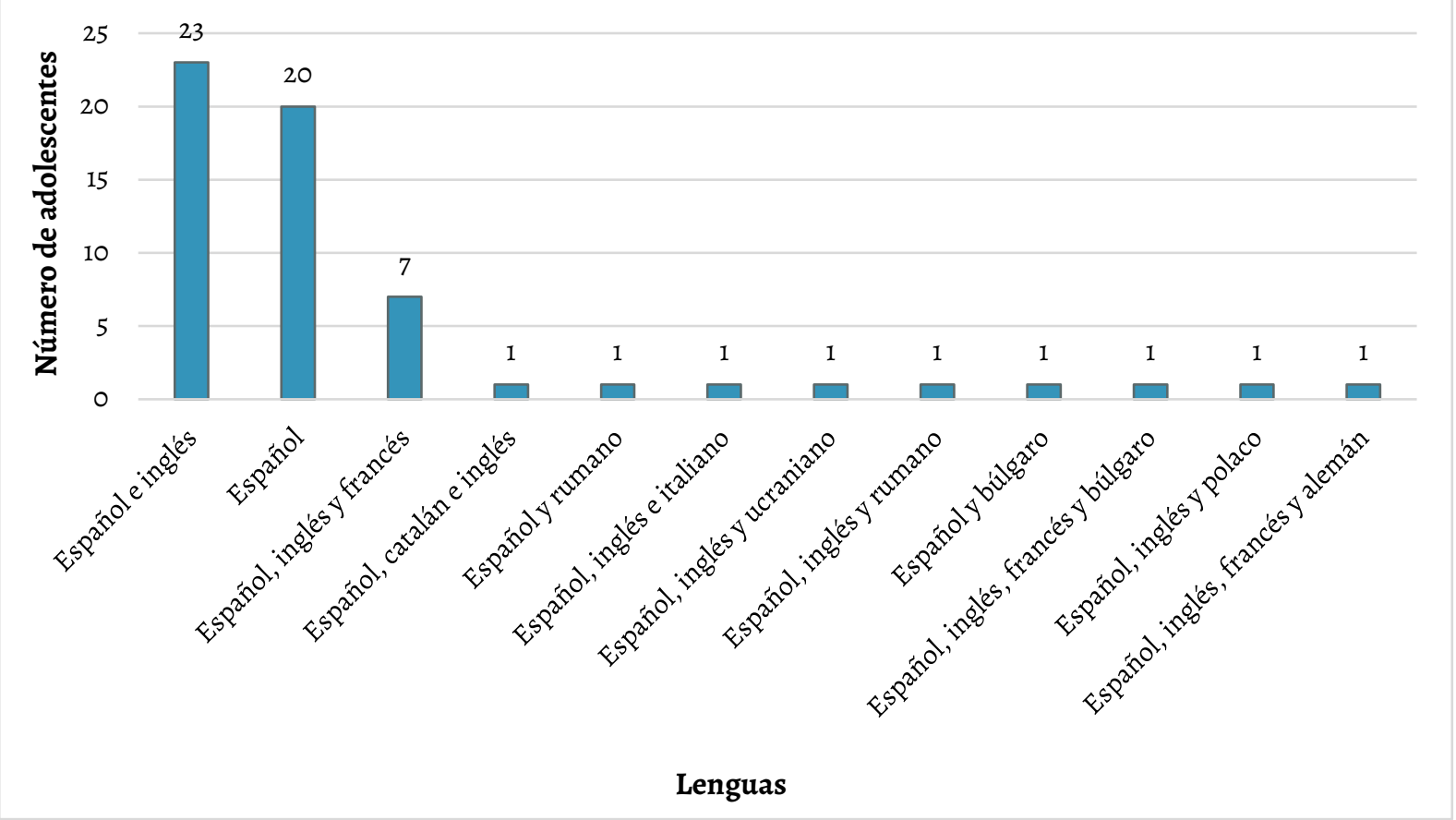

Gráfica 4. Cuestionario para adolescentes. Pregunta: ¿Quélenguas conoces tú? 
Dominio de lenguas de los compañeros

En las gráficas 5 y 6 se muestran las lenguas de los compañeros de los participantes, también muy diversas y numerosas.

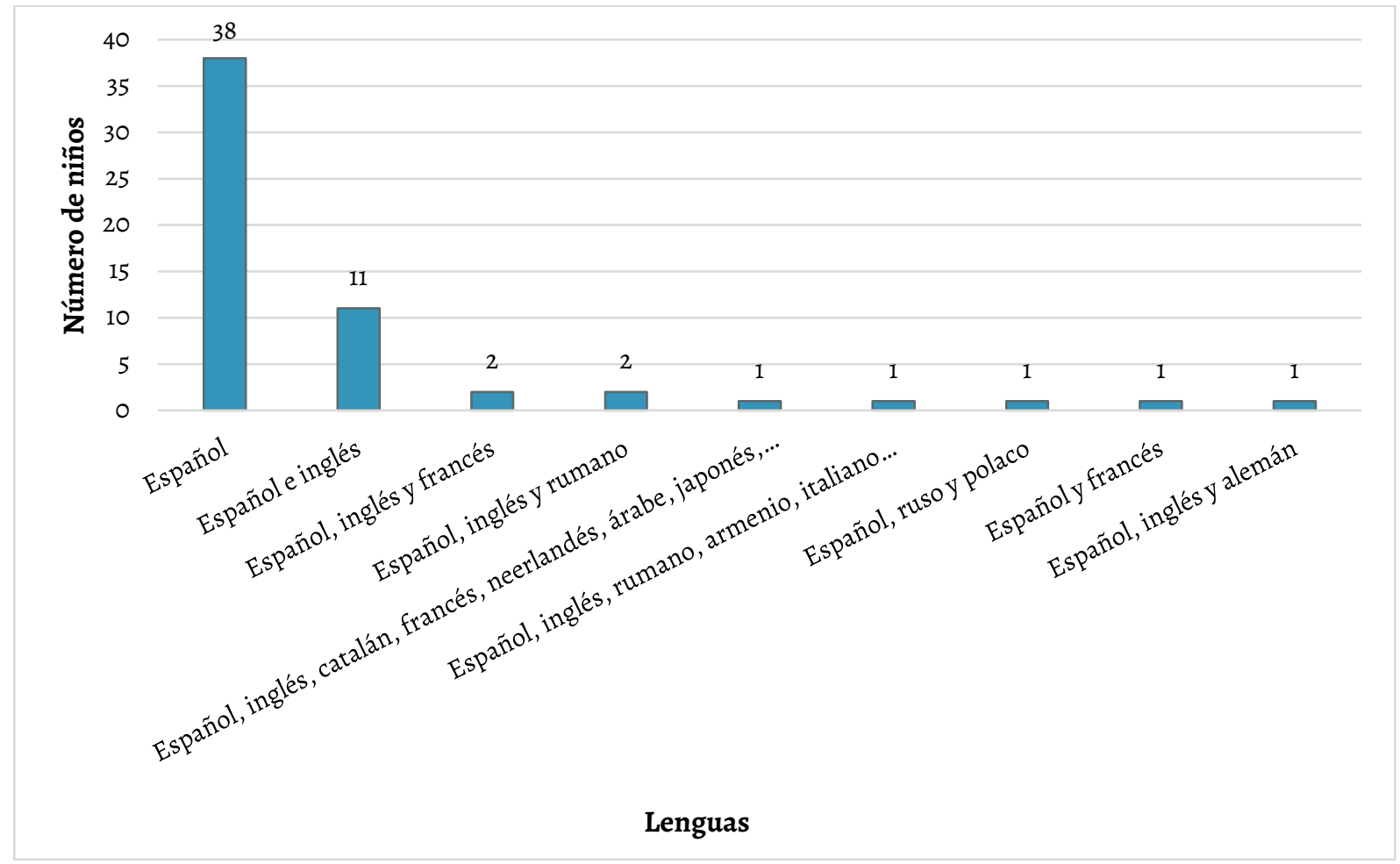

Gráfica 5. Cuestionario para niños. Pregunta: ¿Quélenguas conocen esos compañeros?

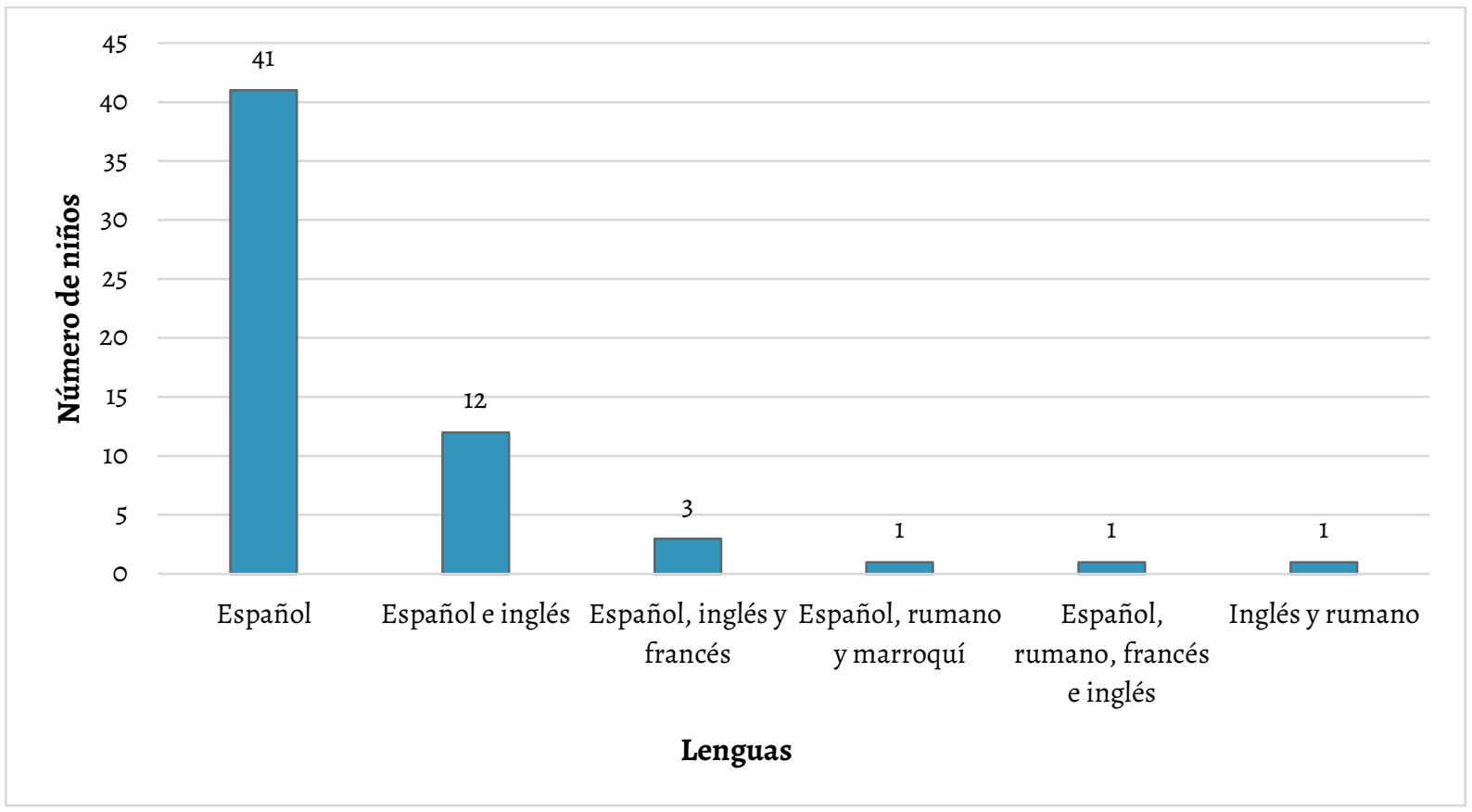

Gráfica 6. Cuestionario para adolescentes. Pregunta: ¿Quélenguas conocen esos compañeros? 
Conviene destacar el ambiente plurilingüe y multicultural en el que conviven los escolares de la zona centro peninsular, resultando ser uno de los principales motivos por los que se ha desarrollado esta iniciativa, que pretende potenciar la relación entre los aprendices de diferentes niveles educativos, incluso durante la reclusión en el hogar. El objetivo de la pregunta que se realizaba a los informantes no es saber el nivel de dominio de las lenguas, sino corroborar la existencia del contacto entre lenguas de diferente origen y la pérdida por parte de los niños y adolescentes del miedo a comunicarse entre iguales, pese a la existencia de grandes diferencias en la competencia lingüística de la lengua materna y/o de la de instrucción o vehicular.

En el siguiente apartado analizamos la fluidez con la que los estudiantes se han relacionado entre sí y a través de qué redes sociales principalmente.

\subsection{Relaciones interpersonales}

La comunicación con sus iguales y las relaciones sociales que se establecen en el ámbito escolar resultan ser aspectos primordiales en la formación de la personalidad del individuo, en la preparación para la convivencia y la integración en la sociedad en la que viven y se desarrollan. Por ello, espacios de intercomunicación que se generan en torno a las aulas, como la realización de tareas en grupos o en parejas, los descansos entre periodos lectivos, los recreos, etc. han resultado especialmente anhelados por los estudiantes de una manera más o menos consciente. Algunos alumnos, integrados en su grupo de iguales, maduros digitalmente y con cierta independencia, han seguido manteniendo relaciones con amigos, compañeros, profesores, etc.; otros han visto bruscamente reducidas sus relaciones fuera del núcleo familiar, y con ello su desarrollo evolutivo en competencia comunicativa.

Analizamos a continuación los datos que nos han proporcionado los informantes de nuestra investigación.

\section{Comunicación entre el alumnado}

Con el fin de conocer si los estudiantes habían mantenido las relaciones con sus iguales, se incorporó en el cuestionario la pregunta ¿Hablas con tus compañeros del cole durante la cuarentena?, que arrojó unos primeros resultados muy positivos puesto que el 93,10\% de los niños y el 94,92\% de los adolescentes respondió afirmativamente. 


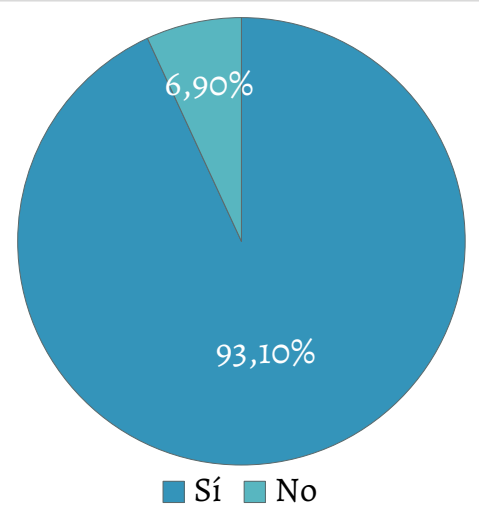

Gráfica 7. Cuestionario para niños. Pregunta: ¿Hablas con tus compañeros del colegio durante la cuarentena?

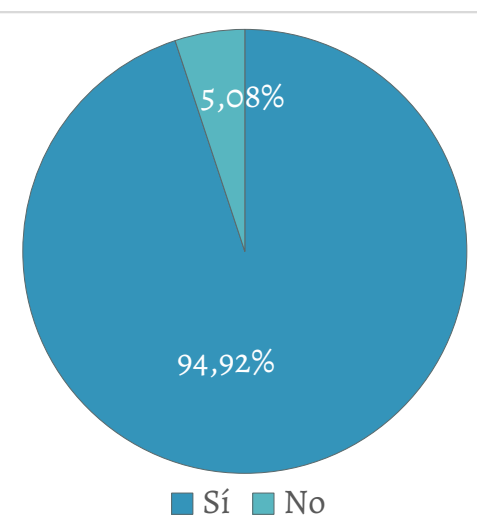

Gráfica 8. Cuestionario para adolescentes. Pregunta: ¿Hablas con tus compañeros del instituto durante la cuarentena?

Los datos recogidos en los cuestionarios cumplimentados por los adolescentes parecen mostrar una mayor interacción entre iguales, o al menos entre los compañeros del centro educativo, pues solo un 5,08\% de los adolescentes que respondieron a la encuesta declaró no hablar con sus compañeros de instituto durante la cuarentena (gráfica 8), aunque es inferior, respecto a los informantes de menor edad, que asciende a casi un $7 \%$. También resulta menor la diversidad lingüística de los amigos declarada por los adolescentes (marroquí y rumano, además de francés e inglés), respecto a la que se registra entre los de menor edad, que mencionan un total de 12 lenguas conocidas por sus compañeros.

Resultados muy semejantes encontramos en una experiencia piloto de implementación del Portfolio Español de las Lenguas (PEL) como instrumento de reflexión multilingüe y multicultural en las aulas de Infantil y de Primaria de dos colegios de Guadalajara y provincia (Fernández López 2013). En las preguntas relacionadas con el conocimiento de lenguas, que sirven de preparación para el uso del PEL con los estudiantes, la totalidad de los alumnos afirmó conocer el español y el inglés; casi un 90\% conocía el francés, 
lengua que en algunos colegios de Guadalajara se incluye entre las materias escolares; y la variedad lingüística y cultural presente en los centros educativos, además de la apertura y disposición de los niños a enfrentarse a nuevas lenguas, hizo que entre un 10 y un $20 \%$ de los alumnos afirmara conocer también el árabe, portugués, italiano y rumano; un $9 \%$, el chino, alguno mencionaba el coreano y el japonés, y un 7\%, el alemán; incluso hacían referencia a otras lenguas peninsulares como el vasco y el catalán, diferenciándolo del valenciano. Al realizar las historias de vida de los estudiantes con los que se trabajaron los PEL muchos aclararon que ellos no hablaban todas esas lenguas, pero las reconocían porque sí las dominaban sus amigos o las habían oído en los medios de comunicación. El dominio de lenguas real de los estudiantes es limitado, pero la predisposición al saber y la pérdida del miedo a no comprender a usuarios de otras lenguas constituyen un importante avance en la inclusión social y la cooperación en el aula.

De la misma manera, en esta nueva experiencia, observando los datos de los niños y adolescentes encuestados, se aprecia que la mayoría hablaba un gran número de lenguas diferentes y se comunicaba con otros compañeros. Sin embargo, sabemos, por la información que nos aportan los docentes que, durante la cuarentena, numerosos niños que se encontraban aprendiendo la lengua vehicular no tuvieron la oportunidad de comunicarse con sus compañeros de clase y solo pudieron hacerlo con sus profesores para entregarles el material correspondiente. Todos los niños y adolescentes que respondieron no a la pregunta ¿Hablas con tus compañeros del colegio/instituto durante la cuarentena? declararon que hablaban español, por lo que no tenemos constancia de que se encontraran en un proceso de aprendizaje del español como segunda lengua.

Redes sociales y plataformas empleadas para la comunicación

Con respecto a la pregunta ¿Qué plataformas o redes sociales utilizas para hablar con ellos?, los niños respondieron con una gran variedad de aplicaciones. La más empleada por ellos fue el WhatsApp, como única aplicación o en combinación con otras. El 68,96\% del total, 40 niños, empleaba el WhatsApp para comunicarse con sus compañeros; 14 niños $(24,13 \%)$ usaban otras aplicaciones diferentes y 4 no utilizaban ninguna (6,89\%), ya que no se comunicaban con sus compañeros durante la cuarentena. 


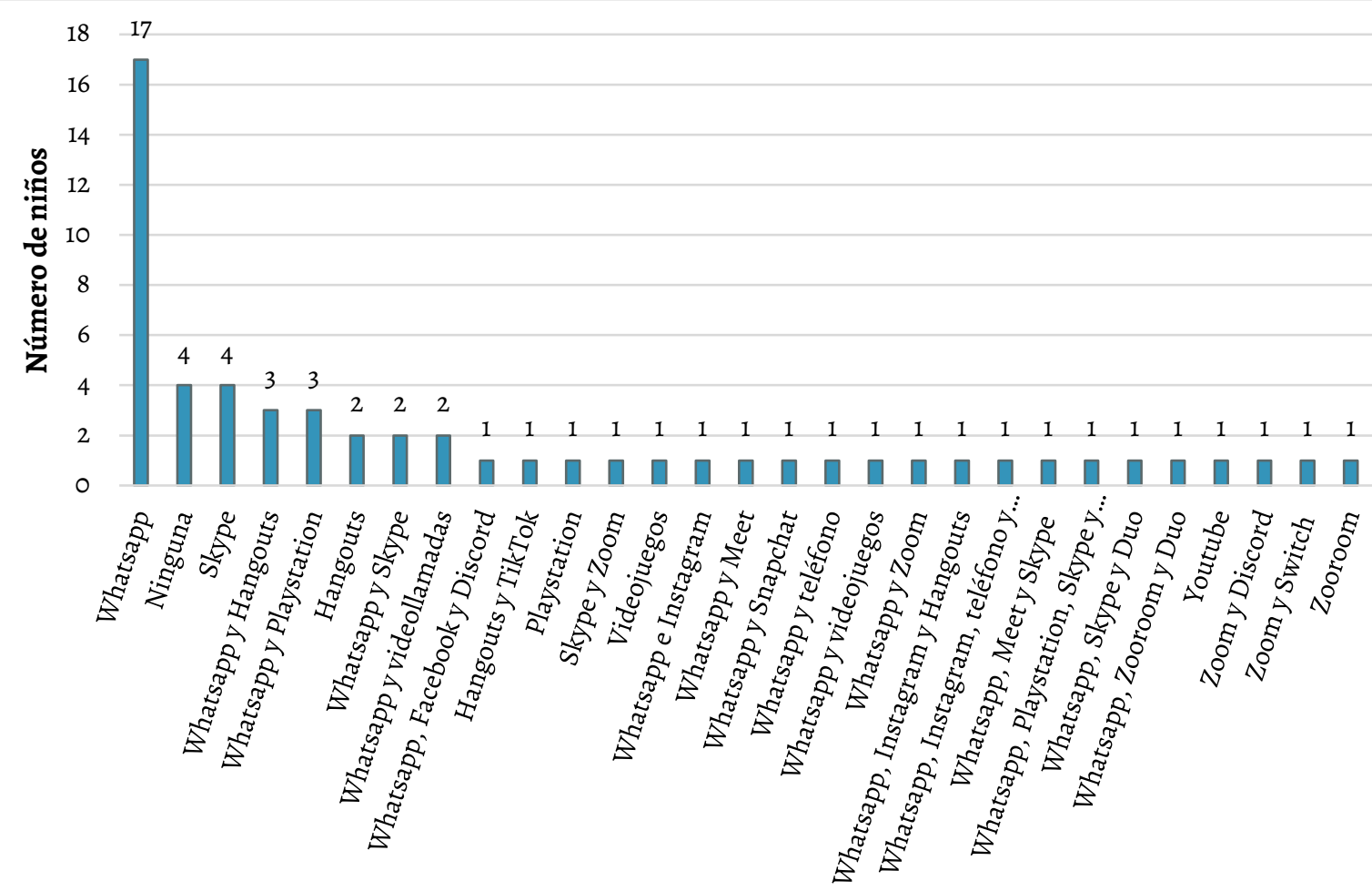

Redes sociales y plataformas

Gráfica 9. Cuestionario para niños. Pregunta: ¿Qué plataformas o redes sociales utilizas para hablar con ellos?

Los adolescentes respondieron con una variedad de aplicaciones menor, como se observa en la gráfica 10. La más empleada por ellos también fue el WhatsApp, como única aplicación o junto a otras (7 la empleaban como única aplicación para comunicarse y 48 la utilizaban junto a otras). Por tanto, 55 adolescentes, que representan un 93,22\% del total, empleaban el WhatsApp para comunicarse con sus compañeros; 1 adolescente solo se comunicaba a través de Instagram $(1,69 \%)$ y 3 no utilizaban ninguna $(5,08 \%)$, ya que no se comunicaban con sus compañeros durante la cuarentena.

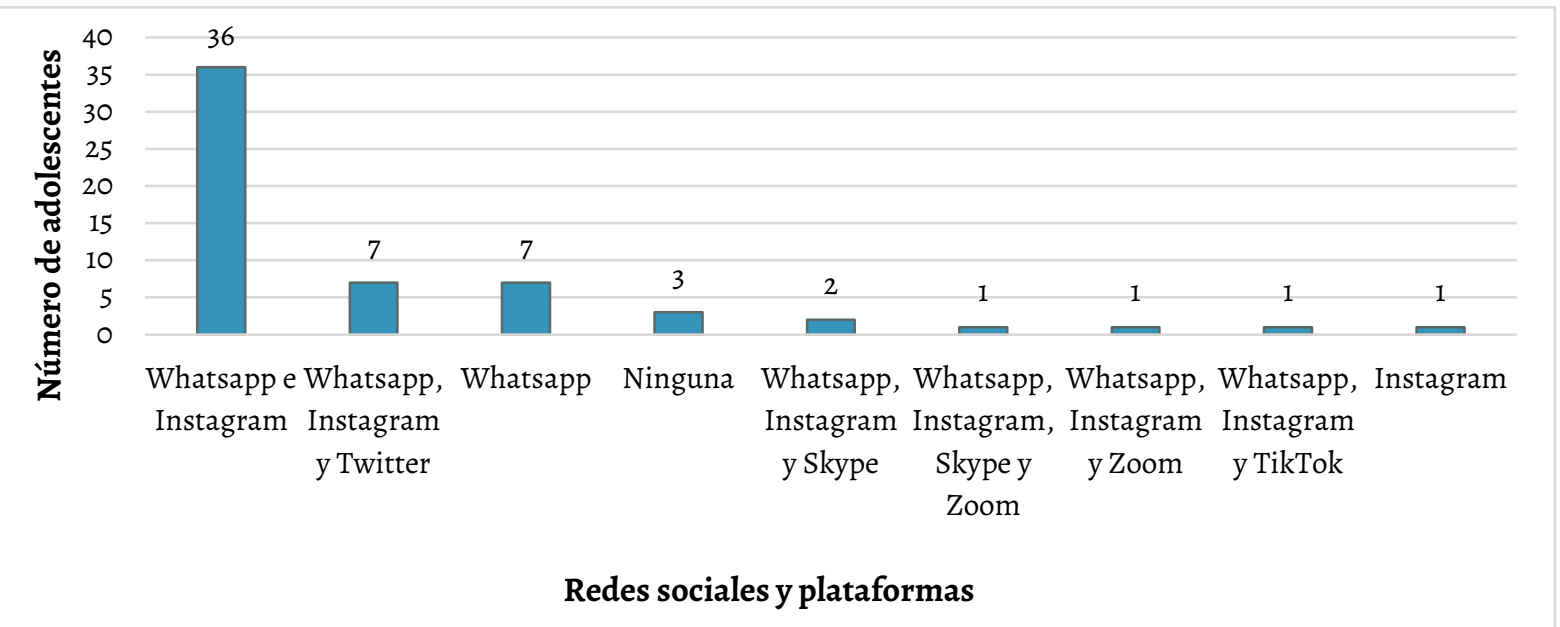

Gráfica 10. Cuestionario para adolescentes. Pregunta: ¿Qué plataformas o redes sociales utilizas para hablar con ellos? 


\subsection{Comunicación escrita}

A continuación, mostramos algunos ejemplos de textos escritos por niños y adolescentes en alguna de las dos opciones que se les presentaban en las preguntas 11 y 12 de cuestionario. Recordamos que los destinatarios de las cartas recibían los textos de los estudiantes que participaron en el estudio, pero con un nuevo formato que se había acordado por las promotoras del proyecto, en el que se incluye el arcoíris, que durante la pandemia se convirtió en un símbolo de ánimo y esperanza. Se reproducen algunos ejemplos anonimizados, por lo que tanto los nombres de los participantes como los datos que puedan resultar identificativos son ficticios. En todos ellos se presentan mensajes de ánimo y muestras de afecto.

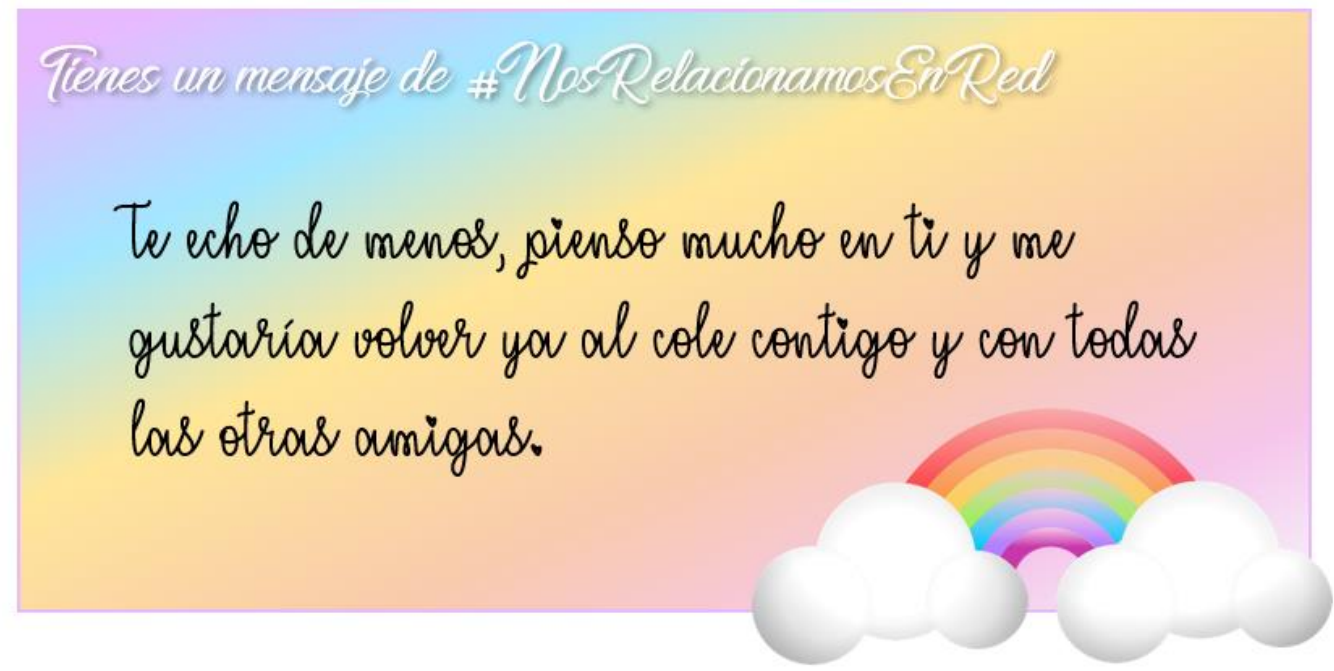

Figura 1. Texto escrito por una niña de 10 años (maquetado y anonimizado según modelo del proyecto).

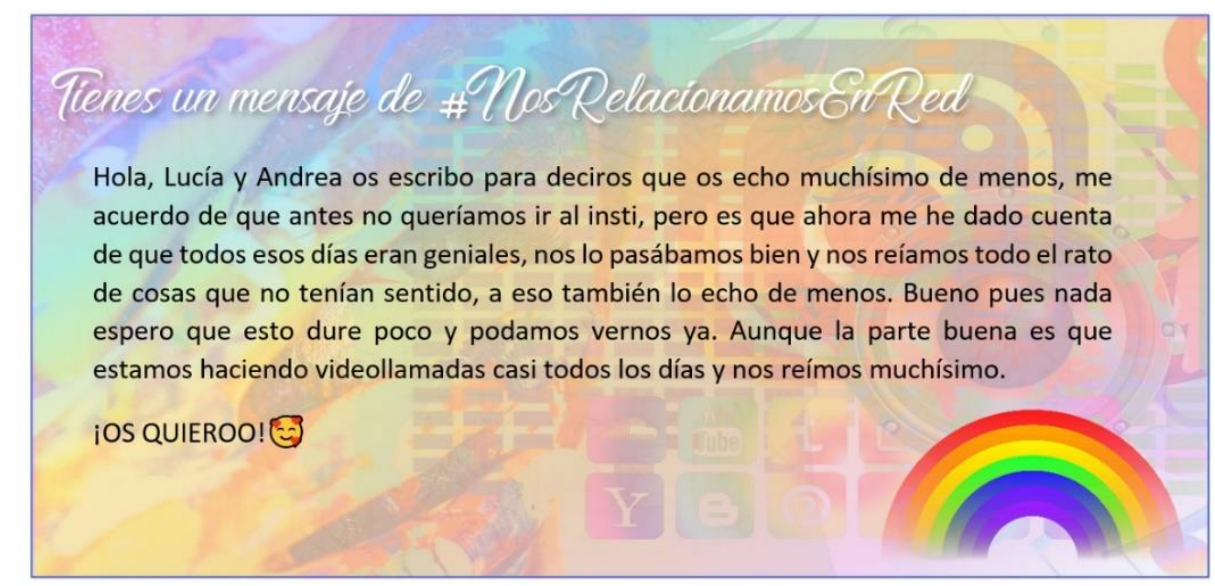

Figura 2. Texto escrito por una adolescente de 16 años (maquetado y anonimizado según modelo del proyecto). 


\section{Tenes un mensaje de \# Mos Relacionamines Ero Red}

Hola Alejandra: te escribo esta carta para decirte que desde que todo esto comenzó no he sabido nada de ti, no me he olvidado de que existes, te mandé un mensaje, pero no lo has visto, tal vez te han vuelto a quitar el móvil (-). Estoy un poco preocupada por no saber nada de ti $\odot$. Espero que tú y tu familia estéis bien, nos veremos pronto, te quiero mucho $O$

Figura 3. Texto escrito por una adolescente de 14 años (maquetado y anonimizado según modelo del proyecto).

\section{Conclusiones y líneas de futuro}

El análisis de los resultados recogidos durante la primera fase nos lleva a continuar investigando qué estudiantes se encontraron aislados durante el confinamiento para buscar propuestas educativas que ayuden a paliar la posible brecha que se hubiera producido en el proceso de integración sociolingüística y cultural de los niños y adolescentes durante el confinamiento en el hogar, y continuar con otras que faciliten el acceso a la lengua en cualquier periodo en el que pueda no haber contacto con el resto de los alumnos como ocurre, por ejemplo, en los periodos de vacaciones de Semana Santa, verano o Navidad.

\section{Referencias bibliográficas}

Barragán Sánchez, R. y E. Ruiz Pinto (2013): "Brecha de género e inclusión digital. El potencial de las redes sociales en educación". Profesorado. Revista de currículum y formación del profesorado, 17, I (enero- abril). https://www.ugr.es/ recfpro/rev171CO L4.pdf

Fernández López, M. C. (2013): "La reflexión sobre las habilidades comunicativas como instrumento para la mejora de la competencia lingüística en Educación Primaria”. En M. C. Sánchez Domínguez y M. Izquierdo Alonso (Dirs.), Delineando lazos hacia nuevas propuestas innovadoras. La interdisciplinaridad como punto de partida, Servicio de Publicaciones de la Universidad de Alcalá, pp. 317-332.

García Navarro, J. (2020): "Niños, adolescentes y redes sociales: Nueva forma de comunicación". Trabajo Final de Grado inédito. Universidad de Alcalá. 
Gobierno de España (2020a): Real Decreto 463/2020, de 14 de marzo, por el que se declara el estado de alarma para la gestión de la situación de crisis sanitaria ocasionada por el COVID-19. https://www.boe.es/eli/es/rd/2020/03/14/463

Gobierno de España (2020b): Real Decreto-ley 10/2020, de 29 de marzo, por el que se regula un permiso retribuido recuperable para las personas trabajadoras por cuenta ajena que no presten servicios esenciales, con el fin de reducir la movilidad de la población en el contexto de la lucha contra el COVID-19.https://www.boe.es/boe/dias/2020/0 3/29/pdfs/BOE-A-2020-4166.pdf

Gobierno de España (2019): Estrategia nacional de prevención y lucha contra la pobreza y la exclusión social (2019-2023). Ministerio de Sanidad, Consumo y Bienestar Social. https://www.eapn.es/ARCHIVO/documentos/noticias/1553262965_estrategia_prev _y_lucha_pobreza_2019-23.pdf

Muñoz Prieto, M. M., M. S. Fragueiro. Barreiro y M. J. Ayuso Manso (2013): "La importancia de las redes sociales en el ámbito educativo", EA, Escuela abierta: revista de Investigación Educativa, (16), pp. 91-104.

Rodicio García, M. L, M. P. Ríos de Deus, M. J. Mosquera González y M. Penado Abilleira (2020). La brecha digital en estudiantes españoles ante la crisis de la Covid-19. Revista internacional de educación para la justicia social (RIEJS), vol. 9, núm. Extra 3: Consecuencias del Cierre de Escuelas por el Covid-19 en las Desigualdades Educativas, pp. 103-125. https://revistas.uam.es/riejs/issue/view/riejs2020_9_3 